\title{
A SPARSITY RESULT ON NONNEGATIVE REAL MATRICES WITH GIVEN SPECTRUM
}

\author{
THOMAS J. LAFFEY \\ Department of Mathematics, University College \\ Belfield, Dublin 4, Ireland \\ E-mail: laffey@acadamh.ucd.ie
}

\begin{abstract}
Let $\sigma=\left(\lambda_{1}, \ldots, \lambda_{n}\right)$ be the spectrum of a nonnegative real $n \times n$ matrix. It is shown that $\sigma$ is the spectrum of a nonnegative real $n \times n$ matrix having at most $(n+1)^{2} / 2-1$ nonzero entries.
\end{abstract}

Let $A=\left(a_{i j}\right)$ be a real $n \times n$ matrix. We say that $A$ is nonnegative if all its entries $a_{i j} \geq 0$ and that $A$ is positive if all $a_{i j}>0$. The nonnegative inverse eigenvalue problem (NIEP) is the problem of characterizing those lists $\sigma=\left(\lambda_{1}, \ldots, \lambda_{n}\right)$ of complex numbers $\lambda_{i}$ for which there exists a nonnegative matrix $A$ with spectrum $\sigma(A)=\sigma$.

If such an $A$ exists we say that the list $\sigma$ is realizable and we say that $A$ realizes $\sigma$. While considerable work has been done on the NIEP, the problem is still far from being solved and in terms of $n$, only in the cases $n=2$ and $n=3$ (Johnson, Loewy and London) has the question been completely settled. See for example [1], [5] for references.

For a given list $\sigma=\left(\lambda_{1}, \ldots, \lambda_{n}\right)$, one can attempt to realize $\sigma$ by the companion matrix $C(f)$ of the polynomial

$$
f(x):=\left(x-\lambda_{1}\right) \cdots\left(x-\lambda_{n}\right):=x^{n}+p_{1} x^{n-1}+\cdots+p_{n} .
$$

In this case $C(f)$ is nonnegative if and only if $p_{i} \leq 0$ for $i=1,2, \ldots, n$.

However this condition is very restrictive - it implies for example that $f(x)$ has only one positive real root (see also [2] for a related discussion) - and one can improve the prospects of success by seeking to realize $\sigma$ by a matrix of the form $\alpha I_{n}+C$ where $\alpha \geq 0$ and $C$ is a nonnegative companion matrix. There exist realizable sets $\sigma$ which are not realizable by matrices of this type. (See Reams' Thesis [6], Chapter 3 for examples with $n=4$.)

1991 Mathematics Subject Classification: Primary 15A48. Secondary 15A33, 15A36.

The paper is in final form and no version of it will be published elsewhere. 
Matrices of the form $\alpha I_{n}+C$ are relatively sparse, having at most $3 n-1$ nonzero entries. This suggests the problem of determining the "sparsest" $n \times n$ matrix realizing a given list $\sigma$ and in this paper, we make a contribution to its resolution.

TheOREM. Suppose $\sigma=\left(\lambda_{1}, \ldots, \lambda_{n}\right)$ is the spectrum of a nonnegative real matrix $B$. Then there exists a nonnegative real $n \times n$ matrix $A$ with spectrum $\sigma$ and such that $A$ has at most $\left[(n+1)^{2} / 2\right]-1$ nonzero entries (where here [ $\left.\cdot\right]$ denotes the greatest integer function).

To prove the theorem, we need the following result.

Lemma. Let $A$ be an $n \times n$ real matrix and suppose that $A$ has $k$ real eigenvalues. Then there exists a subspace $\mathcal{S}$ of $M_{n}(\mathbb{R})$ of dimension $\left(n^{2}-2 n+k\right) / 2$ such that the spectrum $\sigma(A+W)=\sigma(A)$ for all $W \in \mathcal{S}$.

P r o of. By a well-known result of Schur, we can find a real orthogonal matrix $U$ such that $T:=U^{-1} A U$ is in upper block triangular form and where each diagonal block is either a $1 \times 1$ matrix $(a)$ or a $2 \times 2$ matrix $\left(\begin{array}{rr}b & c \\ -c & b\end{array}\right)$ for some real numbers $a, b, c$ with $c \neq 0$, (corresponding to the eigenvalues $a$ or $b \pm i c$ of $A$ ). Furthermore $U$ can be chosen so that the $k$ real eigenvalues of $A$ are $t_{11}, \ldots, t_{k k}$ where $T=\left(t_{i j}\right)$.

Let $\mathcal{S}_{0}$ be the space of all strictly upper-triangular real matrices $B=\left(b_{i j}\right)$ where if $n>k$, so $n-k=2 h$, say, is even, $B$ also has zeros in the positions occurring in the $2 \times 2$ diagonal blocks of $T$ corresponding to nonreal eigenvalues. Thus $b_{i j}=0$ for all $i \leq j$ and also

$$
b_{i j}=0 \quad \text { for }(i, j)=(k+2 l-1, k+2 l), \quad l=1,2, \ldots, h .
$$

Note that if $B \in \mathcal{S}_{0}$, then $T+B$ and $B$ have the same block diagonal and thus $\sigma(A)=$ $\sigma(T)=\sigma(T+B)$.

Note that

$$
\begin{aligned}
\operatorname{dim} \mathcal{S}_{0} & =(n-1)+(n-2)+\ldots+(n-k)+2[(n-k-2)+(n-k-4)+\ldots+2] \\
& =k n-\frac{k(k+1)}{2}+2 h(h-1)=\left(n^{2}-2 n+k\right) / 2 .
\end{aligned}
$$

Defining $\mathcal{S}$ to be $U \mathcal{S}_{0} U^{-1}$, the desired result follows.

Proof of the Theorem. Suppose that $\sigma$ is realizable and let $A$ be a nonnegative matrix which realizes $\sigma$ and subject to this has the greatest possible number of zero entries. Let $\Gamma$ be the set of pairs $(i, j)$ with $a_{i j} \neq 0$. We call $\Gamma$ the support of $A$. Let $\mathcal{M}$ be the span of the matrices $E_{i j}\left((i, j) \in \Gamma\right.$ ) (where $E_{i j}$ is the $n \times n$ matrix with 1 in the $(i, j)$ position, zeros elsewhere).

Since $A$ is nonnegative, the Perron-Frobenius theorem implies that $A$ has at least one real eigenvalue, so, by the Lemma, there is a subspace $\mathcal{S}$ of $M_{n}(\mathbb{R})$ of dimension at least $\left(n^{2}-2 n+1\right) / 2$ such that $\sigma(A+W)=\sigma(A)$ for all $W \in \mathcal{S}$.

Claim. $\mathcal{S} \cap \mathcal{M}=\{0\}$.

For, if not, let $0 \neq B \in \mathcal{S} \cap \mathcal{M}$. Since the support of $B$ is contained in the support of $A, A+a B$ is nonnegative and has the same support as $A$ for all sufficiently small $a$ and thus we can choose $b$ such that $A+b B$ is nonnegative and such that $(A+b B)$ has its 
$(i, j)$ entry 0 for some $(i, j) \in \Gamma$. But since $\sigma(A+b B)=\sigma(A)$, this contradicts our choice of $A$. So the claim holds.

Now $\mathcal{S}+\mathcal{M}$ is a subspace of $M_{n}(\mathbb{R})$ so

$$
\operatorname{dim} \mathcal{M} \leq n^{2}-\operatorname{dim} \mathcal{S} \leq\left(n^{2}+2 n-1\right) / 2=(n+1)^{2} / 2-1 .
$$

This proves the Theorem.

Corollary. Suppose $\sigma=\left(\lambda_{1}, \ldots, \lambda_{n}\right)$ is the spectrum of a nonnegative matrix $B$. Then $\sigma$ is the spectrum of an $n \times n$ nonnegative matrix $A$ having at least $n-1$ of its entries equal to 0 .

\section{Remarks}

1. One can show that $\left(n^{2}+n\right) / 2$ is in fact the correct bound in the theorem for $n=2,3$. For $n=4$, the bound in the theorem is 11 but we do not know an example requiring more than 9 nonzero entries.

2. If

$$
\sum_{i=1}^{n} \lambda_{i}=0
$$

we can replace $\mathcal{M}$ in the above proof by $\mathcal{M}_{0}:=\mathcal{M}+\operatorname{span}\left\{E_{11}\right\}$ and the claim holds because (in the notation of the proof of the theorem)

$$
\mathcal{S} \cap \mathcal{M}_{0}=\mathcal{S} \cap \mathcal{M}
$$

since the elements of $\mathcal{S}$ have trace 0 . So in the trace 0 case, the bound can be improved by 1 .

3. Suppose that $\sigma=\left(\lambda_{1}, \ldots, \lambda_{n}\right)$ is realizable and that $A=\left(a_{i j}\right)$ realizes $\sigma$. We define

$$
s_{k}:=\operatorname{trace}\left(A^{k}\right)=\lambda_{1}^{k}+\cdots+\lambda_{n}^{k} .
$$

If $A$ has exactly $m$ nonzero entries on the diagonal an argument independently constructed by Johnson [3] and Loewy and London [4] shows that

$$
m^{k-1} s_{k} \geq s_{1}^{k}
$$

So, in particular, if $(n-1) s_{2}<s_{1}^{2}, A$ must have all its diagonal entries different from 0 . Furthermore if the digraph of $A$ has no 2-cycles (that is $a_{i j} a_{j i} \neq 0$ for all $i, j$ with $i \neq j$ ) then it is an easy exercise to check that $s_{1} s_{3} \geq s_{2}^{2}$. More generally, if $r>1$ is the smallest integer $r$ for which the digraph of $A$ contains an $r$-cycle (that is, there exist distinct integers $j_{1}, j_{2}, \ldots, j_{r}$ such that $a_{j_{1} j_{2}} a_{j_{2} j_{3}} \cdots a_{j_{r-1} j_{r}} a_{j_{r} j_{1}} \neq 0$ ), then

$$
s_{k}=a_{11}^{k}+\cdots+a_{n n}^{k} \quad \text { for } k=1,2, \ldots, r-1
$$

and

$$
s_{r}>a_{11}^{r}+\cdots+a_{n n}^{r}
$$

and thus

$$
s_{k} s_{l} \geq s_{p} s_{q}
$$

for all positive integers $k, l, p, q$ with

$$
k \leq p \leq q \leq l \quad \text { and } \quad k+l=p+q \leq r .
$$


Thus we may use inequalities between the $s_{i}$ to get lower bounds on the size of the support of $A$. In this way, one can construct examples to show that the bound in the theorem is of the right order of magnitude for $n=2,3$ and 4 . For large values of $n$, however, this author does not know any example of a realizable spectrum of size $n$ which is not realizable by a matrix with at most $4 n+1$ nonzero entries. So it is tempting to conjecture that the best possible bound in the theorem is linear rather than quadratic in $n$.

4. We say that $\sigma=\left(\lambda_{1}, \ldots, \lambda_{n}\right)$ is an extreme spectrum if $\sigma$ is realizable but for all $\alpha>0,\left(\lambda_{1}-\alpha, \ldots, \lambda_{n}-\alpha\right)$ is not realizable. One can show that solving the NIEP is equivalent to characterizing extreme spectra. Suppose $A \geq 0$ realizes an extreme spectrum $\sigma$. If $A$ is reducible under permutation similarity, that is, there exists a permutation matrix $P$ such that

$$
P^{-1} A P=\left(\begin{array}{cc}
A_{11} & A_{12} \\
0 & A_{22}
\end{array}\right)
$$

where $A_{11}$ is $r \times r, A_{22}$ is $(n-r) \times(n-r)$ for some $r$ with $1 \leq r<n$, then $\sigma(A)=$ $\sigma\left(A_{11}\right) \cup \sigma\left(A_{22}\right)$ and $A_{11}$ and $A_{22}$ have smaller size and an inductive argument can be invoked.

So we can assume $A$ is irreducible.

In this case, the Perron-Frobenius theorem states that $A$ has an eigenvector $v$ corresponding to the positive eigenvalue $\rho$ which that $v$ has all its entries positive. Replacing $A$ by $D^{-1} A D$ for a positive diagonal matrix, we can assume $v=j$, the vector of all 'ones'. Suppose $A$ has a column with all entries positive. We assume column one of $A$ has strictly positive entries. Choose $\epsilon_{1}<0, \epsilon_{2}>0, \ldots, \epsilon_{n}>0$ with $\sum_{i=1}^{n} \epsilon_{i}=0$ and such that

$$
A_{\epsilon}=A+\left(\epsilon_{1} j \epsilon_{2} j \cdots \epsilon_{n} j\right)>0 .
$$

But $A_{\epsilon}$ has the same spectrum as $A$.

Since $\sigma$ is extreme, this is impossible.

Hence $A$ has at least one zero entry in each column and similarly in each row. Suppose now that $\operatorname{trace}(A)>0$. Let $\Gamma=\operatorname{supp}(A)$, the support of $A$, and let $X=\left(x_{i k}\right)$ have zero entries off $\Gamma$ and indeterminate entries $x_{i k}(i, k) \in \Gamma$. Consider the system of equations

$$
\operatorname{trace}\left(A^{r} X\right)=n \rho^{r} \quad \text { for } r=0,1,2, \ldots,(n-1) .
$$

If this system is solvable, then

$$
\operatorname{trace}\left(A^{r}(J-X)\right)=0 \quad \text { for } r=0,1,2, \ldots, n-1
$$

(where $J$ is the matrix with all entries equal to 1 ). Thus if $A$ is nonderogatory (so in particular, if $A$ has distinct eigenvalues), then

$$
J-X=[A, T]=A T-T A
$$

for some matrix $T$.

Consider for small $\epsilon>0$,

$$
A_{\epsilon}=(I+\epsilon T)^{-1} A(1+\epsilon T)=A+\epsilon[A, T]+O\left(\epsilon^{2}\right)=A+\epsilon(J-X)+O\left(\epsilon^{2}\right) .
$$

Since $\operatorname{supp}(X) \subseteq \operatorname{supp}(A), A+\epsilon(J-X)$ has positive entries for small $\epsilon>0$. So $A_{\epsilon}>0$ for all sufficiently small $\epsilon>0$. But $\sigma\left(A_{\epsilon}\right)=\sigma(A)$ is extreme. This is a contradiction. 
So the system $(*)$ is inconsistent.

Since $(*)$ contains as many indeterminates as the support of $A$, this inconsistency is particularly restrictive if $A$ is not relatively sparse. The argument shows that the only "generic" class of extreme spectra is the class of those with $s_{1}=0$, that is, the spectra of trace zero nonnegative matrices.

\section{References}

[1] M. Boyle, Symbolic dynamics and matrices, in: Combinatorial and Graph-Theoretical Problems in Linear Algebra (eds. Brualdi, Friedland and Klee), IMA Vol. Math. Appl. 50 (1993), 1-38.

[2] D. Handelman, Spectral radii of primitive integral companion matrices and log concave polynomials, in: Symbolic dynamics and its applications, Contemp. Math. 135 (1992), 231-238.

[3] C. R. Johnson, Row stochastic matrices that are similar to doubly stochastic matrices, Linear and Multilinear Algebra 10 (1981), 113-120.

[4] R. Loewy and D. London, A note on the inverse problem for nonnegative matrices, Linear and Multilinear Algebra 6 (1978), 83-90.

[5] T. J. Laffey, Inverse eigenvalue problem for matrices, to appear in Hamilton Conference Proceedings, Royal Irish Academy.

[6] R. Reams, Topics in Matrix Theory, Thesis presented for the degree of Ph.D., National University of Ireland, 1994. 